\title{
Exact asymptotic expansion for the resistance between the center node and a node on the cobweb network boundary
}

\author{
N. Izmailian 112, R. Kenna $a^{21}$ \\ 1 Yerevan Physics Institute, Alikhanian Brothers 2, 375036 Yerevan, Armenia \\ 2 Applied Mathematics Research Center, Coventry University, Coventry CV1 5FB, England
}

Received May 4, 2014, in final form June 2, 2014

\begin{abstract}
We analyze the resistance between two nodes in a cobweb network of resistors. Based on an exact expression, we derive the asymptotic expansions for the resistance between the center node and a node on the boundary of the $M \times N$ cobweb network with resistors $r$ and $s$ in the two spatial directions. All coefficients in this expansion are expressed through analytical functions.
\end{abstract}

Key words: resistor network, asymptotic expansion

PACS: $05.50 .+q, 05.60 . C d, 02.30 . \mathrm{Mv}$

\section{Introduction}

The classic problem in electrical circuit theory, first studied by Kirchhoff in 1847, is the calculation of the resistance between two arbitrary nodes in a resistor network [1]. Besides its long-standing importance in electric circuit theory, the computation of resistances is also connected to a wide range of problems as diverse as random walks [2, 3], first-passage processes [4], lattice Green's functions [5] and classical transport in disorder media [6-8].

In $2004 \mathrm{Wu}$ [9] derived a closed-form expression for the two-point resistance in terms of the eigenvalues and eigenvectors of the Laplacian matrix associated with the network. Quite recently, Izmailian, Kenna and $\mathrm{Wu}$ [10] revisited the problem of two-point resistance and derived a new and simpler expression for the resistance between two arbitrary nodes for finite networks with resistors $r$ and $s$ in the two spatial directions. The new expression was then applied to the cobweb resistor network [10].

Essam and $\mathrm{Wu}$ [11] used one of the results [9] to derive the asymptotic expansion for the corner-tocorner resistance $R(r, s)$ on an $M \times N$ rectangular resistor network under free boundary conditions. This was extended by Izmailian and Huang [12] to other boundary conditions. In recent decades, the finitesize scaling and finite-size corrections in finite critical systems and their boundary effects have attracted much attention [11-30]. Of particular importance in such studies are exact results wherein the analysis can be carried out without numerical errors.

In this paper we derive the exact asymptotic expansion for the resistance between the central node and a node on the boundary of the cobweb network. We show that this expansion can be written in the form

$$
\frac{1}{s} R(r, s)=c(h) \ln S+c_{0}(h, \xi)+\sum_{p=1}^{\infty} \frac{c_{2 p}(h, \xi)}{S^{p}}
$$

with $h=s / r, S=(M+1 / 2) N$ and $\xi=(M+1 / 2) / N$. Note that, instead of the actual length $M$, we have used effective length $(M+1 / 2)$. All coefficients in this expansion $\left[c(h), c_{0}(h, \xi), c_{2 p}(h, \xi)\right]$ are expressed through analytical functions. The computation of the asymptotic expansion of the resistance between two 
maximally separated nodes of a rectangular resistor network has been of interest for some time, because its value provides a lower bound to the resistance of compact percolation clusters in the Domany-Kinzel model of a directed percolation [31].

The organization of this paper is as follows. Based on the exact expression for the resistance between two arbitrary nodes for a finite cobweb resistor network obtained in [10] we express the resistance between the central node and a node on the boundary of the network in terms of $G_{\alpha, \beta}(h, M, N)$ with $(\alpha, \beta)=(0,1 / 2)$ (section 2). We then extend the algorithm of Ivashkevich, Izmailian and Hu [13] to derive exact asymptotic expansions for the resistance between the central node and a node on the boundary of the cobweb resistor network and write down the expansion coefficients (section 3). Finally, we discuss our results in section 4.

\section{Two-dimensional resistor networks}

The resistor network can be regarded as a graph consisting of $T$ nodes and let $R_{i, j}=R_{j, i}$ be the resistance of the resistor connecting the nodes $i$ and $j$. Denote the nonzero eigenvalues and eigenvectors of the Laplacian of that network by $\lambda_{i}$ and $\Psi_{\mathbf{i}}=\left(\psi_{i 1}, \psi_{i 2}, \ldots, \psi_{i T}\right)$, respectively. Then, the resistance between the nodes $i$ and $j$ can be written as [9]

$$
R_{i, j}=\sum_{k=2}^{T} \frac{\left|\psi_{k i}-\psi_{k j}\right|^{2}}{\lambda_{k}} .
$$

Let us consider the cobweb network. The cobweb lattice $\mathscr{L}_{\text {cob }}$ is an $M \times N$ rectangular lattice with periodic boundary conditions in one direction and nodes on one of the two boundaries in the other direction connected to an external common node. Therefore, there is a total of $M N+1$ nodes. The example of an $M=3, N=8$ cobweb with resistors $s$ and $r$ in the two directions is shown in figure 1 . Topologically $\mathscr{L}_{\text {cob }}$ is of the form of a wheel consisting of $N$ spokes and $M$ concentric circles. We use the term DirichletNeumann to describe the boundary conditions along the innermost apex and outermost arc. There has been a considerable recent interest in studying the resistance in a cobweb network (see for example [10, 32, 33]).

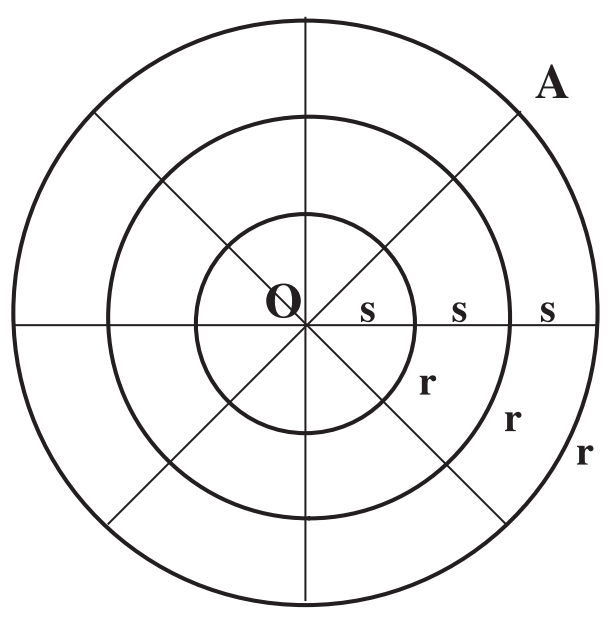

Figure 1. An $M \times N$ cobweb network with $M=3$ and $N=8$. Bonds in the radial and circular directions comprise resistors $s$ and $r$. The center point is denoted by $O, A$ denotes any point on the boundary of the cobweb network.

The closed-form expression for the resistance $R\left(\mathbf{r}_{1}, \mathbf{r}_{2}\right)$ between two arbitrary nodes $\mathbf{r}_{1}=\left(x_{1}, y_{1}\right)$ and $\mathbf{r}_{2}=\left(x_{2}, y_{2}\right)$ for the cobweb network was obtained in [10]. In what follows, we will show that the resistance $R(O, A)$ between the central node $O=(0,0)$ and a node on the boundary of the network $A=(x, M)$ 
can be expressed in terms of $G_{0,1 / 2}(\mathscr{M}, \mathscr{N})$ only,

$$
R^{\mathrm{cob}}(0, A)=-\frac{s}{2}+\frac{\sqrt{s r}}{4 S} G_{0,1 / 2}(2 M+1, N)
$$

where $S=(M+1 / 2) N$ and $G_{\alpha, \beta}(\mathscr{M}, \mathscr{N})$ is given by

$$
G_{\alpha, \beta}(\mathscr{M}, \mathscr{N})=\mathscr{M} \operatorname{Re} \sum_{n=0}^{\mathcal{N}-1} f\left(\pi \frac{n+\alpha}{\mathscr{N}}\right) \operatorname{coth}\left[\mathscr{M} \omega\left(\pi \frac{n+\alpha}{\mathscr{N}}\right)+\mathrm{i} \pi \beta\right],
$$

for $(\alpha, \beta) \neq(0,0)$. The function $\omega(y)$ is given by:

$$
\omega(y)=\operatorname{arcsinh} \sqrt{h} \sin y
$$

and the function $f(y)$ is given by

$$
f(y)=\frac{\sqrt{1+h \sin ^{2} y}}{\sin y} .
$$

\subsection{Cobweb network}

The resistance between the central node $O=(0,0)$ and the other node $C=(x, y)$ of the cobweb network is given by (see second line of equation (33) of [10])

$$
R^{\mathrm{cob}}(O, C)=\frac{2 s}{N(2 M+1)} \sum_{m=0}^{M-1} \sum_{n=0}^{N-1} \frac{\sin ^{2}\left(2 y \varphi_{m}\right)}{h\left(1-\cos 2 \theta_{n}\right)+\left(1-\cos 2 \varphi_{m}\right)}, \quad y=1,2, \ldots, M,
$$

where $h=s / r$ and

$$
\theta_{n}=\frac{\pi n}{N}, \quad \varphi_{m}=\frac{\pi(m+1 / 2)}{2 M+1} .
$$

Note that the result (2.6) is independent of the position $x$ as it should be.

In the special case of the resistance between the center $O$ and a point $A=\{x, N\}$ on the outer boundary of the cobweb network, we use $y=M$ and obtain from (2.6)

$$
R^{\mathrm{cob}}(O, A)=\frac{s}{N(2 M+1)} \sum_{m=0}^{M-1} \sum_{n=0}^{N-1} \frac{\cos ^{2} \varphi_{m}}{\sin ^{2} \varphi_{m}+h \sin ^{2} \theta_{n}},
$$

where use has been made of the identity

$$
\sin \left(2 M \varphi_{m}\right)=(-1)^{m} \cos \varphi_{m},
$$

which is a consequence of the fact $2 M \varphi_{m}+\varphi_{m}=\left(m+\frac{1}{2}\right) \pi$.

Equation (2.8) can be transformed as follows:

$$
\begin{aligned}
R^{\mathrm{cob}}(O, A) & =\frac{s}{N(2 M+1)} \sum_{m=0}^{M-1} \sum_{n=0}^{N-1}\left[-1+\frac{1+h \sin ^{2} \theta_{n}}{\sin ^{2} \varphi_{m}+h \sin ^{2} \theta_{n}}\right], \\
& =-\frac{s M}{2 M+1}+\frac{s}{N(2 M+1)} \sum_{m=0}^{M-1} \sum_{n=0}^{N-1} \frac{1+h \sin ^{2} \theta_{n}}{\sin ^{2} \varphi_{m}+h \sin ^{2} \theta_{n}} .
\end{aligned}
$$

We can extend the summation over $m$ in equation (2.11) from $M-1$ up to $2 M$ and obtain the expression

$$
R^{\mathrm{cob}}(O, A)=-\frac{s}{2}+\frac{s}{2 N(2 M+1)} \sum_{m=0}^{2 M} \sum_{n=0}^{N-1} \frac{1+h \sin ^{2} \theta_{n}}{\sin ^{2} \varphi_{m}+h \sin ^{2} \theta_{n}} .
$$

The sum over $m$ in the equation (2.12) can be carried out using the identity [12]

$$
\sum_{m=0}^{\mathscr{M}-1}\left[h \sin ^{2} \theta_{n}+\sin ^{2} \frac{\pi\left(m+\frac{1}{2}\right)}{\mathscr{M}}\right]^{-1}=2 \mathscr{M} \frac{\operatorname{coth}\left[\mathscr{M} \omega\left(\theta_{n}\right)+\mathrm{i} \pi / 2\right]}{\sinh 2 \omega\left(\theta_{\mathrm{n}}\right)},
$$


with $\mathscr{M}=2 M+1$ and $\omega(x)$ given by equation (2.4). It is easy to see that

$$
1+h \sin ^{2} \theta_{n}=\cosh ^{2} \omega\left(\theta_{n}\right)
$$

Plugging equations (2.13) and (2.14) back in equation (2.12) we obtain that $R^{\operatorname{cob}}(O, A)$ can be written in the form

$$
R^{\operatorname{cob}}(O, A)=-\frac{s}{2}+\frac{s}{2 N} \sum_{n=0}^{N-1} \frac{\operatorname{coth}\left[(2 M+1) \omega\left(\theta_{n}\right)+\mathrm{i} \pi / 2\right]}{\tanh \omega\left(\theta_{\mathrm{n}}\right)}
$$

Using identity

$$
\tanh \omega\left(\theta_{n}\right)=\frac{\sqrt{h} \sin \theta_{n}}{\sqrt{1+h \sin ^{2} \theta_{n}}},
$$

equation (2.15) can be finally written in the form given by equation (2.2).

\section{Asymptotic expansion}

In section 2 we have shown that the resistance between the central node and a node on the boundary of the cobweb resistor network can be expressed in terms of the function $G_{0,1 / 2}(x, \mathcal{M}, \mathscr{N})$ only, [see equations [2.2]]. Using the method proposed in [13], Izmailian and Huang [12] derived the asymptotic expansion of $G_{\alpha, \beta}(\mathscr{M}, \mathscr{N})$ in terms of the so-called Kronecker double series [34], which are directly related to elliptic $\theta$ functions. We next need the asymptotic expansion of $G_{0,1 / 2}(\mathscr{M}, \mathscr{N})$, which can be found in Appendix A

After reaching this point, one can easily write down all the terms of the exact asymptotic expansion for the resistance between the central node and a node on the boundary of the cobweb network $\left[R^{\mathrm{cob}}(O, A)\right]$ using equations $[2.2$ and $(\mathrm{A} .1)$. We have found that the exact asymptotic expansion of the $R^{\mathrm{cob}}(O, A)$ can be written as equation (1.1).

\subsection{Asymptotic expansion for the resistance between the central node and a node on the boundary of the cobweb network}

For the cobweb network we obtain

$$
\begin{aligned}
\frac{1}{s} R^{\mathrm{cob}}(O, A)= & \frac{1}{2 \pi \sqrt{h}}\left[\ln S+2 \ln \frac{8}{\pi}+2 C_{\mathrm{E}}-1-\ln \xi(1+h)+2 \sqrt{h} \arctan \sqrt{h}-4 \ln \theta_{2}(2 \mathrm{i} \sqrt{h} \xi)\right] \\
& -\frac{1}{2 \pi \sqrt{h}} \sum_{p=1}^{\infty}\left(\frac{\pi^{2} \xi}{S}\right)^{p} \frac{\Omega_{2 p}}{p(2 p) !} \mathrm{K}_{2 p}^{0,1 / 2}(2 \mathrm{i} \sqrt{h} \xi) .
\end{aligned}
$$

Thus, the coefficients $c_{2 p}(h, \xi)(p=1,2, \ldots)$ in the expansion (1.1) are explicitly given by

$$
c_{2 p}(h, \xi)=-\frac{\pi^{2 p-1} \xi^{p}}{2 p(2 p) ! \sqrt{h}} \Omega_{2 p} \mathrm{~K}_{2 p}^{0,1 / 2}(2 \mathrm{i} \sqrt{h} \xi),
$$

where the differential operators $\Omega_{2 p}$ are given by equation (A.2) and $K_{2 p}^{0,1 / 2}(2 \mathrm{i} \sqrt{h} \xi$ ) is Kronecker's double series which can all be expressed in terms of the elliptic $\theta_{k}(2 i \sqrt{h} \xi)(k=2,3,4)$ functions only.

Here, we list the first few coefficients in the expansion given by equation (1.1):

$$
\begin{aligned}
c(h) & =\frac{1}{2 \pi \sqrt{h}} \\
c_{0}(h, \xi) & =\frac{1}{2 \pi \sqrt{h}}\left[2 \ln \frac{8}{\pi}+2 C_{\mathrm{E}}-1-\ln \xi(1+h)+2 \sqrt{h} \arctan \sqrt{h}-4 \ln \theta_{2}\right], \\
c_{2}(h, \xi) & =\frac{\pi \tau_{0}}{288 h}\left\{(1+3 h)\left(\theta_{3}^{4}+\theta_{4}^{4}\right)+2 \tau_{0}(1+h)\left[\pi \theta_{3}^{4} \theta_{4}^{4}+2\left(\theta_{3}^{4}+\theta_{4}^{4}\right) \frac{\partial}{\partial \tau_{0}} \ln \theta_{2}\right]\right\}
\end{aligned}
$$


To simplify the notation we have used the short hand

$$
\theta_{k}=\theta_{k}\left(\mathrm{i} \tau_{0}\right), \quad k=2,3,4
$$

where $\tau_{0}=2 \xi \sqrt{h}$.

We have also used the following relations between derivatives of the elliptic functions:

$$
\frac{\partial}{\partial \tau_{0}} \ln \theta_{3}=\frac{\pi}{4} \theta_{4}^{4}+\frac{\partial}{\partial \tau_{0}} \ln \theta_{2} \quad \text { and } \quad \frac{\partial}{\partial \tau_{0}} \ln \theta_{4}=\frac{\pi}{4} \theta_{3}^{4}+\frac{\partial}{\partial \tau_{0}} \ln \theta_{2} .
$$

Note that elliptic functions $\theta_{2}, \theta_{3}, \theta_{4}$ can be expressed through the complete elliptic integral of the first kind $K=K(k)$ and second kind $E=E(k)$ as follows:

$$
\theta_{2}=\sqrt{\frac{2 k K(k)}{\pi}}, \quad \theta_{3}=\sqrt{\frac{2 K(k)}{\pi}}, \quad \theta_{4}=\sqrt{\frac{2 k^{\prime} K(k)}{\pi}}
$$

where

$$
\begin{aligned}
& K(k)=\int_{0}^{\pi / 2} \frac{\mathrm{d} x}{\sqrt{1-k^{2} \sin ^{2} x}}, \\
& E(k)=\int_{0}^{\pi / 2} \sqrt{1-k^{2} \sin ^{2} x} \mathrm{~d} x .
\end{aligned}
$$

With the help of the identities

$$
\frac{\partial}{\partial \tau_{0}} \ln \theta_{2}=-\frac{1}{2} \theta_{3}^{2} E, \quad \text { and } \quad \frac{\partial E}{\partial \tau_{0}}=\frac{\pi^{2}}{4} \theta_{3}^{2} \theta_{4}^{4}-\frac{\pi}{2} \theta_{4}^{4} E
$$

one can express all derivatives of the elliptic functions in terms of the elliptic functions $\theta_{2}, \theta_{3}, \theta_{4}$ and the complete elliptic integral of the second kind $E=E(k)$.

Thus, we have obtained explicit analytic formulas for all corrections to scaling terms $c_{2 p}(h, \xi)$ in the form of elliptic functions. For the case $\xi=1$ and $h=1$, we have the following results:

$$
\frac{1}{s} R^{\mathrm{cob}}(O, A)=\frac{1}{\pi} \ln N+c_{0}+\frac{c_{2}}{N^{2}}+\ldots
$$

with $c_{0}=0.9286495235004523 \ldots$ and $c_{2}=0.3572873939981 \ldots$.

\section{Discussion}

In the present paper, we study the two-point resistor problem on the cobweb network. Using the exact expression for the resistance between two arbitrary nodes for a finite cobweb network obtained in [10] and the IIH's algorithm [13], we derive the exact asymptotic expansion of the resistance between the central node and a node on the boundary of the cobweb resistor networks. All coefficients in this expansion are expressed through analytical functions.

\section{Acknowledgements}

The work was supported by a Marie Curie IIF (Project no. 300206-RAVEN) and IRSES (Projects no. 295302-SPIDER and 612707-DIONICOS) within 7th European Community Framework Programme and by the grant of the Science Committee of the Ministry of Science and Education of the Republic of Armenia under contract 13-1C080. 


\section{A. Asymptotic expansion of $G_{0,1 / 2}(2 M+1, N)$}

The asymptotic expansion of $G_{\alpha, \beta}(\mathscr{M}, \mathscr{N})$ for $(\alpha, \beta) \neq(0,0)$ has been obtained in [12]. Here, we will reproduce the result of the paper [12] for the case $(\alpha, \beta)=(0,1 / 2), \mathscr{M}=2 M+1$ and $\mathscr{N}=N$. After little algebra, the asymptotic expansion of $G_{0,1 / 2}(2 M+1, N)$ can be written as follows:

$$
\begin{aligned}
G_{0,1 / 2}(2 M+1, N)= & \frac{4 S}{\pi}\left[\frac{1}{2} \ln \frac{S}{\xi}+C_{\mathrm{E}}+\ln \frac{8}{\pi}-\frac{1}{2} \ln (1+h)+\sqrt{h} \arctan \sqrt{h}-2 \ln \left|\theta_{2}(2 \mathrm{i} \xi \sqrt{h})\right|\right] \\
& -2 \pi \xi \sum_{p=1}^{\infty}\left(\frac{\pi^{2} \xi}{S}\right)^{p-1} \frac{\Omega_{2 p}}{p(2 p) !} \operatorname{Re~K}_{2 p}^{0,1 / 2}(2 \mathrm{i} \xi \sqrt{h}),
\end{aligned}
$$

where $S=(M+1 / 2) N, \xi=(M+1 / 2) / N, C_{\mathrm{E}}$ is the Euler constant, $\theta_{2}(\tau)$ is elliptic theta function and $K_{2 p+2}^{0,1 / 2}(\tau)$ is Kronecker's double series [34].

The differential operators $\Omega_{2 p}$ that have appeared here can be expressed via coefficients $\omega_{2 p}=\varepsilon_{2 p}+$ $\lambda_{2 p} \frac{\partial}{\partial \lambda}$ as

$$
\begin{aligned}
& \Omega_{2}=\omega_{2}, \\
& \Omega_{4}=\omega_{4}+3 \omega_{2}^{2},
\end{aligned}
$$

where $\lambda_{2 p}$ and $\kappa_{2 p}$ are the coefficients in the Taylor expansion of $\omega(y)$, given by equation (2.4) and $f(y)$ given by equation (2.5), respectively

$$
\omega(y)=y\left[\lambda+\sum_{p=1}^{\infty} \frac{\lambda_{2 p}}{(2 p) !} y^{2 p}\right]
$$

with $\lambda=\sqrt{h}, \lambda_{2}=-\frac{1}{3} \sqrt{h}(1+h), \lambda_{4}=\frac{1}{5} \sqrt{h}\left(1+10 h+9 h^{2}\right)$, etc., and

$$
f(y)=\frac{1}{y}\left[1+\sum_{p=1}^{\infty} \frac{\kappa_{2 p}}{(2 p) !} y^{2 p}\right],
$$

with $\kappa_{2}=-\frac{1}{3}-h, \kappa_{4}=-\frac{7}{15}+2 h+3 h^{2}$, etc. Note that function $f(y)$ can be represented as

$$
f(y)=\frac{1}{y} \exp \left\{\sum_{p=1}^{\infty} \frac{\varepsilon_{2 p}}{(2 p) !} y^{2 p}\right\},
$$

and the coefficients $\varepsilon_{2 p}$ and $\kappa_{2 p}$ are related to each other through the relation between moments and cumulants

$$
\begin{aligned}
& \kappa_{2}=\varepsilon_{2}, \\
& \kappa_{4}=\varepsilon_{4}+3 \varepsilon_{2}^{2},
\end{aligned}
$$

The Kronecker's double series $K_{2 p}^{0,1 / 2}(\tau)$ can all be expressed in terms of the elliptic $\theta(\tau)$ functions only. Equations for $K_{2 p}^{0,1 / 2}(\tau)$ with $p=2,3,4,5$ and other useful relations for elliptic $\theta$-functions and Kronecker's double series can be found in [13-16]. 


\section{References}

1. Kirchhoff G., Ann. Phys. Chem., 1847, 72, 497; doi 10.1002/andp.18471481202

2. Doyle P.G., Snell J.L., Random Walks and Electric Network, The Carus Mathematical Monograph, series 22, The Mathematical Association of America, USA, 1984, pp. 83-149.

3. Lovász L., Random Walks on Graphs: A Survey in Combinatorics, Paul Erdöis Eighty Vol. 2, Miklós D., Sós V.T., Szónyi T. (Eds.), Janos Bolyai Mathematical Society, Budepest, 1996, pp. 353-398.

4. Redner S., A Guide to First-Passage Processes, Cambridge University Press, Cambridge, 2001.

5. Katsura S., Morita T., Inawashiro S., Horiguchi T., Abe Y., J. Math. Phys., 1971, 12, 892; doi 10.1063/1.1665662

6. Harris A.B., Lubensky T.C., Phys. Rev. B, 1987, 35, 6964; doi 10.1103/PhysRevB.35.6964

7. Kirkpatrick S., Rev. Mod. Phys., 1973, 45, 574; doi 10.1103/RevModPhys.45.574.

8. Derrida B., Vannimenus J., J. Phys. A: Math. Gen., 1982, 15, L557; doi 10.1088/0305-4470/15/10/007

9. Wu F.Y., J. Phys. A: Math. Gen., 2004, 37, 6653; doi 10.1088/0305-4470/37/26/004

10. Izmailian N.Sh., Kenna R., Wu F.Y., J. Phys. A: Math. Theor., 2014, 47, 035003; doi 10.1088/1751-8113/47/3/035003

11. Essam J.W., Wu F.Y., J. Phys. A: Math. Theor., 2009, 42, 025205; doi 10.1088/1751-8113/42/2/025205

12. Izmailian N.Sh., Huang M.-C., Phys. Rev. E, 2010, 82, 011125; doi 10.1103/PhysRevE.82.011125

13. Ivashkevich E.V., Izmailian N.Sh., Hu C.-K., J. Phys. A: Math. Gen., 2002, 35, 5543; doi 10.1088/0305-4470/35/27/302

14. Izmailian N.Sh., Oganesyan K.B., Hu C.-K., Phys. Rev. E, 2002, 65, 056132; doi 10.1103/PhysRevE.65.056132

15. Izmailian N.Sh., Oganesyan K.B., Hu C.-K., Phys. Rev. E, 2003, 67, 066114; doi 10.1103/PhysRevE.67.066114

16. Izmailian N.Sh., Hu C.-K., Phys. Rev. E, 2007, 76, 041118; doi 10.1103/PhysRevE.76.041118

17. Blote H.W.J., Cardy J.L., Nightingale M.P., Phys. Rev. Lett., 1986, 56, 742; doi 10.1103/PhysRevLett.56.742

18. Cardy J.L., Nucl. Phys. B, 1986, 275, 200; doi 10.1016/0550-3213(86)90596-1

19. Hu C.-K., Chen J.A., Izmailian N.Sh., Kleban P., Phys. Rev. E, 1999, 60, 6491; doi 10.1103/PhysRevE.60.6491.

20. Izmailian N.Sh., Hu C.-K., Phys. Rev. Lett., 2001, 86, 5160; doi 10.1103/PhysRevLett.86.5160

21. Kaneda K., Okabe Y., Phys. Rev. Lett., 2001, 86, 2134; doi 10.1103/PhysRevLett.86.2134

22. Lu W.T., Wu F.Y., Phys. Rev. E, 2001, 63, 026107; doi 10.1103/PhysRevE.63.026107

23. Janke W., Kenna R., Phys. Rev. B, 2002, 65, 064110; doi 10.1103/PhysRevB.65.064110.

24. Izmailian N.Sh., Hu C.-K., Phys Rev. E, 2002, 65, 036103; doi 10.1103/PhysRevE.65.036103

25. Wu M.-C., Chin-Kun Hu C.-K., Izmailian N.Sh., Phys. Rev. E, 2003, 67, 065103(R); doi 10.1103/PhysRevE.67.065103

26. Izmailian N.Sh., Priezzhev V.B., Ruelle P., Hu C.-K., Phys. Rev. Lett., 2005, 95, 260602; doi $10.1103 /$ PhysRevLett.95.260602

27. Izmailian N.Sh., Oganesyan K.B., Wu M.-C., Hu C.-K., Phys. Rev. E, 2006, 73, 016128; doi 10.1103/PhysRevE.73.016128

28. Izmailian N.Sh., Priezzhev V.B., Ruelle P., Symmetry Integr. Geom., 2007, 3, 001; doi 10.3842/SIGMA.2007.001

29. Izmailian N.Sh., Yeh Y.-N., Nucl. Phys. B, 2009, 814, 573; doi 10.1016/j.nuclphysb.2009.01.017

30. Izmailian N.Sh., Hu C.-K., Nucl. Phys. B, 2009, 808, 613; doi 10.1016/j.nuclphysb.2008.09.009

31. Domany E., Kinzel W., Phys. Rev. Lett., 1984, 53, 311; doi 10.1103/PhysRevLett.53.311

32. Essam J.W., Tan Z.-Z., Wu-F.Y., Preprint arXiv:1312.6727 2013.

33. Tan Z.-Z., Zhou L., Yang J.-H., J. Phys. A: Math. Theor., 2013, 46, 195202; doi 10.1088/1751-8113/46/19/195202

34. Weil A., Elliptic functions according to Eisenshtein and Kronecker, Springer-Verlag, Berlin-Heidelberg-New York, 1976. 


\title{
Точний асимптотичний розклад для опору між
} центральним вузлом і вузлом на границі павутинної мережі

\author{
Н. Ізмаілян 112, Р. Кенна
}

1 єреванський фізичний інститут, м. Єреван, Вірменія

2 Центр прикладних математичних досліджень, університет м. Ковентрі, м. Ковентрі, Англія

Ми аналізуємо опір між двома вузлами в павутинній мережі резисторів. На основі точного виразу ми виводимо асимптотичні розклади для опору між центральним вузлом і вузлом на границі павутинної мережі $M \times N$ з резисторами $r$ і $s$ у двох просторових напрямках. Всі коефіцієнти в цьому розкладі виражаються через аналітичні функції.

Ключові слова: резисторна мережа, асимптотичний розклад 